left colon $(n=20)$ and rectum $(n=7)$. Compared to ER performed by trainers, there were no differences in failure to achieve endoscopic clearance $(p=0.09)$, complications $(p=0.67)$, intraprocedural bleeding $(p=0.32)$ or recurrence $(p=0.42)$.

Conclusions These results show that, in a specialist unit with experienced trainers, it is safe to train residents in complex EMR/pEMR who can perform the techniques safely in large CSNL with good outcomes. Significant prior experience in diagnostic colonoscopy is important but the skills for advanced assessment and EMR can subsequently be acquired in the right setting.

\section{PTH-073 RADIOLOGICAL STAGING INVESTIGATIONS BEFORE ENDOSCOPIC RESECTION OF LARGE COLORECTAL LESIONS: SIGNIFICANT BURDEN WITH NO BENEFIT}

Andrew Emmanuel, Shraddha Gulati, Monica Ortenzi, Margaret Burt, Bu Hayee, Amyn Haji. King's College Hospital, London, UK

\subsection{6/gutjnl-2018-BSGAbstracts.94}

Introduction Endoscopic resection (ER) is increasingly used for curative treatment of large colorectal superficial neoplastic lesions (CSNL). Experts believe that accurate lesion assessment and in vivo diagnosis should guide treatment decisions for such lesions, however in western practice skills in lesion assessment are less robust and patients frequently undergo biopsy sampling and pre-procedure radiological staging investigations as for any suspected colorectal cancer. For large rectal lesions, many consider pelvic MRI obligatory. The value of such investigations in this context is not clear.

Methods All ER of large $(\geq 20 \mathrm{~mm})$ CSNLs referred to a tertiary unit were included. Data was collected from clinical letters, endoscopy and radiology reports from the referring department as well as the lesion assessment, treatment and final histopathology at our institution. Details of the findings of computed tomography (CT) scans, need for subsequent imaging and potential staging of rectal tumours by MRI were recorded.

Results 579 CSNLs $\geq 20 \mathrm{~mm}$ were treated with ER. 177 patients $(31 \%)$ had received a staging CT of the thorax, abdomen and pelvis prior to referral. Of 163 rectal tumours, 67 (41\%) had received a staging MRI. The findings of the CT scan did not change the management of the CSNL in any patients. Incidental findings were reported in 28 patients $(16 \%)$. As a result 25 (89\%) went on to require further imaging or referral to other clinicians which resulted in treatment for only one patient who required a ureteric stent for an asymptomatic obstructing stone. No MRI was reported as less than T1 and $31(30 \%)$ were reported as at least T2 or greater, of which only 3 had invasive adenocarcinoma: 2 were T1 with minimal submucosal invasion and one was recognised during the ER as having deep invasion but was unfit for surgery. MRIs in 10 patients were reported as N1-2 (positive lymph node metastases), only 3 had proven adenocarcinoma of which only one eventually agreed to surgery: there was a T3N2 adenocarcinoma but surgery was performed more than 3 years after the initial MRI.

Conclusions Traditional staging radiological investigations have no value in the management of either colonic or rectal large CSNL assessed as likely non-invasive using endoscopic assessment. Instead, they are a significant burden on resources, expose patients to unnecessary radiation, are likely to contribute to unfounded increased anxiety for patients and clinicians and lead to a significant number of additional investigations or specialist consultations without meaningful outcome.

\section{PTH-074 SMSA SCORE NOT AN INDEPENDENT PREDICTOR OF OUTCOMES IN A LARGE SERIES OF ENDOSCOPIC RESECTIONS}

Andrew Emmanuel, Shraddha Gulati, Monica Ortenzi, Margaret Burt, Bu Hayee, Amyn Haji. King's College Hospital, London, UK

\subsection{6/gutjnl-2018-BSGAbstracts.95}

Introduction A predictive score to stratify the difficulty of endoscopic resection (ER) of large colorectal superficial neoplastic lesions (CSNL) and predict outcomes would be valuable. The SMSA score assigns a numerical score based on size, morphology, site and ease of access and stratifies lesions into 4 groups of increasing complexity. It has been recommended in several guidelines on colorectal ER and SMSA level 4 is reportedly associated with incomplete resection and increased complications. Despite this, it has not been widely validated in large series of ER for large CSNLs using standardised techniques. We applied the SMSA score to a large series of ER of lesions $\geq 2 \mathrm{~cm}$ at a tertiary centre.

Methods ER of large $(\geq 2 \mathrm{~cm})$ CSNL were included. Surveillance colonoscopy was performed at 3 months and 12 months. SMSA score was calculated and SMSA level 4 compared with level 2-3 for outcomes including failed ER, $\geq 2$ ER to achieve clearance, complications and recurrence. Multivariate logistic regression was performed to determine independent predictors of complications and recurrence.

Results ER was performed for 542 lesions (mean size $53.7 \mathrm{~mm}$ ). SMSA level 4 was not associated with an increased risk of failed ER (OR $0.06,95 \%$ CI $0.007-0.44, \mathrm{p}<0.001$ ) or requirement of $\geq 2$ ER to achieve clearance (OR 3.96, 95\% CI 0.49-31.9, $\mathrm{p}=0.16$ ). Although SMSA level 4 was associated with complications (OR 5.25, 95\% CI 1.58-17.5, p=0.002), it was not associated with perforation and a simple assessment of either large lesion size $(\geq 50 \mathrm{~mm})$ or difficult access was similarly associated with complications (OR 4.27, 95\% CI 1.47-12.4, $\mathrm{p}=0.004)$. Multivariate logistic regression revealed only age $(\mathrm{p}<0.001)$, prior heavy manipulation $(\mathrm{p}=0.03)$, and ESD/Hybrid ESD $(p=0.02)$ were independently associated with complications but not SMSA level $4(p=0.06)$.

SMSA level 4 was associated with recurrence (OR 10.8, 95\% CI 2.59-45.2, p<0.001), as was either large lesion size or difficult access (OR 6.35, 95\% CI 2.47-16.33, p<0.001). On multivariate logistic regression only large size/difficult access $(p=0.03)$ and piecemeal resection $(p=0.003)$ were associated with recurrence but not SMSA level 4.

Conclusion SMSA level 4 was not associated with failed ER or multiple ERs to achieve clearance. It was not an independent predictor of complications or recurrence on multivariate logistic regression. Although relatively simple, the SMSA score is somewhat difficult to rapidly calculate and stratify lesions. For practical purposes when making a judgement during a procedure, it may be as useful to use a simple assessment of large lesion size or difficulty in accessing the lesion. 